\title{
SWAP: Sun Watcher using APS detector on-board PROBA-2, a new EUV off-axis telescope on a technology demonstration platform
}

\author{
J.M. Defise ${ }^{1 *}$, D. Berghmans ${ }^{2}$, J.F. Hochedez ${ }^{2}$, J.H. Lecat ${ }^{1}$, E. Mazy ${ }^{1}$, P. Rochus ${ }^{1}$, T. Thibert ${ }^{1}$, \\ P. Nicolosi ${ }^{3}$, M.G. Pelizzo ${ }^{3}$, U. Schühle ${ }^{4}$, R. A. M. Van der Linden ${ }^{2}$, A. N. Zhukov ${ }^{2}$ \\ ${ }^{1}$ Centre Spatial de Liège - Av. Pré Aily B-4031 Angleur - Belgium \\ ${ }^{2}$ Royal Observatory of Belgium - Ringlaan- 3 -Av. Circulaire, B-1180 Brussels- Belgium \\ ${ }^{3}$ INFM-LUXOR, Dept of Information Engineering, University of Padova \\ ${ }^{4}$ Max Planck Institut für Aeronomie - Lindau - Germany
}

\begin{abstract}
SWAP (Sun Watcher using Active Pixel System detector and Image Processing) is an instrument that has been selected to fly on the PROBA-II technology demonstration platform, a program of the European Space Agency (ESA) to be launched in 2006. This paper presents the instrument concept and its scientific goals.

SWAP uses an off-axis Ritchey Chretien telescope that will image the EUV solar corona at $19.5 \mathrm{~nm}$ on a specifically fabricated extreme ultraviolet (EUV) sensitivity enhanced CMOS APS detector. This type of detector has advantages that promise to be very profitable for solar EUV imaging. The SWAP design is built on a similar concept as the MAGRITTE instrument ${ }^{1,2}$ suite for the NASA Solar Dynamics Observatory (SDO)mission to be launched in 2007. The optics have been adapted to the detector size. The SWAP PROBA-2 program will be an opportunity to demonstrate and validate the optical concept of MAGRITTE, while it will also validate space remote sensing with APS detectors.

On the science outcomes, SWAP will provide solar corona images in the Fe XII line on a baselined 1-min cadence. Observations with this specific wavelength allow detecting phenomena, such as solar flares or 'EIT-waves', associated with the early phase of coronal mass ejections. Image recognition software will be developed that automatically detects these phenomena and sends out space weather warnings. Different modules of this software will run both on the ground system as well as on the onboard computer of PROBA II. The SWAP data will complement the observations provided by SOHO-EIT, and STEREO-SECCHI.
\end{abstract}

Keywords: Solar corona, EUV telescopes, PROBA

\section{INTRODUCTION TO PROBA-2}

The PROBA-2 small satellite (> $120 \mathrm{~kg}$ ), to be developed under an ESA General Support Technology Program (GSTP) contract by a consortium led by Verhaert Design \& Development (Belgium) has two main mission objectives: (i) perform an in-flight demonstration of a series of new spacecraft technologies and (ii) support a scientific mission of a set of selected instruments. As a follow on of PROBA- $1^{3}$ successfully in orbit since October 2001, the performances and the autonomous functions previously demonstrated by PROBA-1 will also be met or exceeded.

The technology demonstrations are in the field of avionics, spacecraft attitude control, power system and spacecraft propulsion. Two instruments are dedicated to the observation of the Sun, SWAP (imaging instrument) and LYRA (radiometer instrument), and two others are dedicated to plasma measurements. Possibly an Earth observation instrument will be included as well.

${ }^{*}$ Correspondence - email: jmdefise@ulg.ac.be, phone: +32 4367 6668, fax: +3243675613 
Figures 1 and 2 show the preliminary accommodation concept of PROBA-2, with LYRA and SWAP emerging on the sun side of the payload.

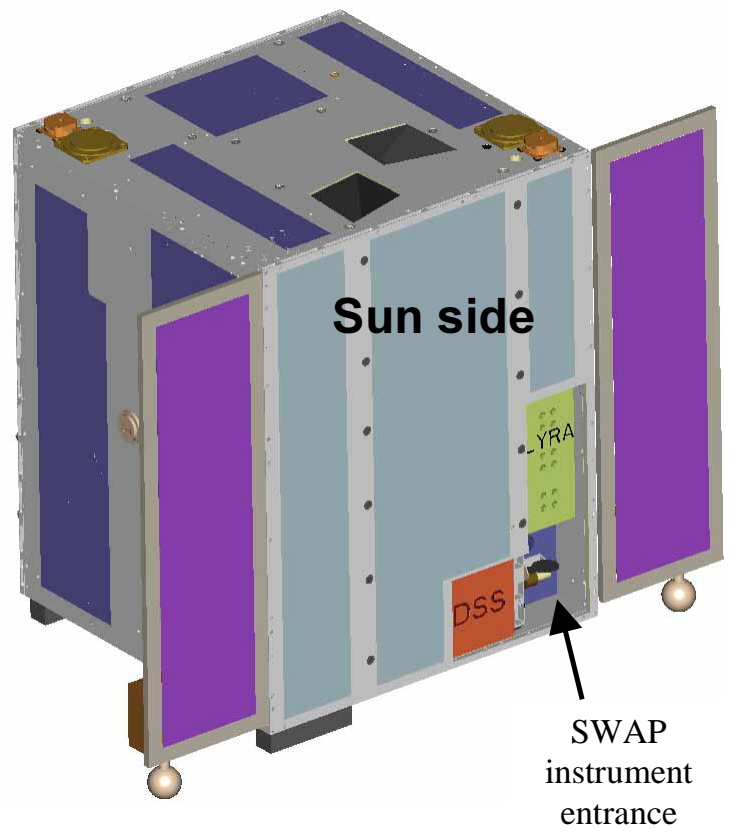

Figure 1: View of the Proba-2 spacecraft

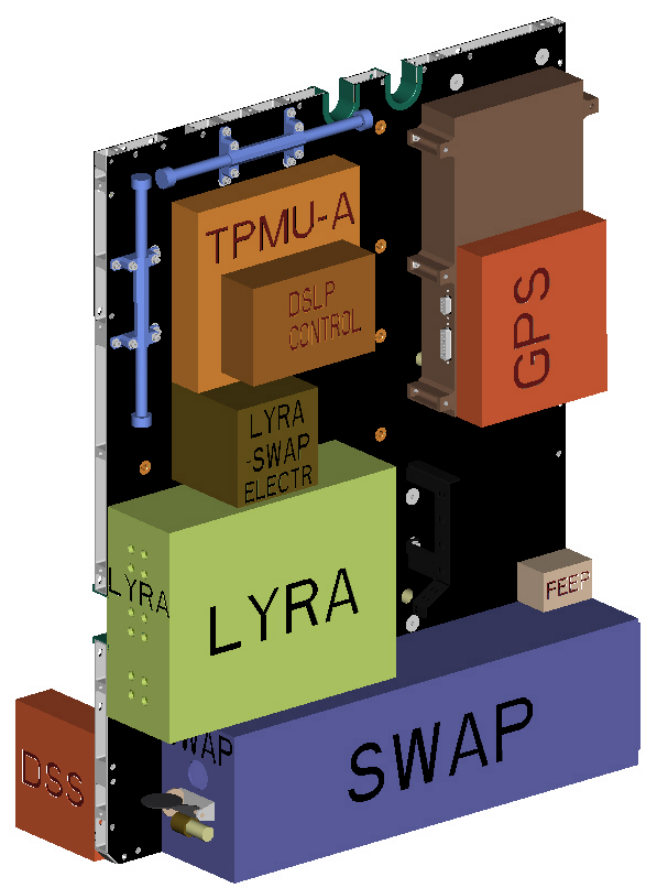

Figure 2: PROBA-2 Payload (part)

\section{SWAP OBSERVATIONS AND SPACE WEATHER APPLICATIONS}

SWAP builds upon the heritage of the Extreme ultraviolet Imaging Telescope ${ }^{4}$ (EIT) onboard the joint ESA-NASA mission SOHO. The latter monitors the solar corona since 1996. In its nominal 'CME watch program', EIT takes an image in its $19.5 \mathrm{~nm}$ bandpass every $15 \mathrm{~min}$. The EIT instrument has proved to be particularly useful for space weather monitoring. A study ${ }^{5}$ on the European space weather assets conducted by the Rutherford Appleton Laboratory on behalf of ESA came to the conclusion that "the main European contribution to current space weather monitoring is ESA's SOHO mission, in particular through the Images from the LASCO and EIT instrument" .

The $19.5 \mathrm{~nm}$ bandpass that SWAP will inherit from EIT gives indeed a particularly broad view on the 'solar weather'. Single $19.5 \mathrm{~nm}$ images already give important information on the location of coronal holes (the source of high speed solar wind streams), active regions (potential source of solar flares) and filaments (potential eruption sites). Following the temporal evolution of these features as they rotate, gives additional important inputs to space weather forecasters.

Yet, accurate monitoring is only achieved by having high cadence time sequence of $19.5 \mathrm{~nm}$ images. Such sequences give information on more subtle events like EIT waves (faint global waves propagating across the solar disc from the CME eruption site ${ }^{6}$ ), coronal EUV dimming regions (transient coronal holes from where the CME material has lifted off) and filament instabilities (a specific type of flickering during the rise of a filament that could serve as a CME precursor). Systematic detection by the SWAP instrument of these phenomena that are associated with the early development of CMEs allows to pinpoint the source region of coronal mass ejections and let differentiate between back-sided and front-sided halo CMEs. The accurate idenfication of the last type of CMEs gives a 3-day warning before the CME can hit the Earth magnetosphere and cause geomagnetic storms.

SWAP will continue the systematic CME watch program of the aging EIT instrument, but at an image cadence (1 image every minute instead of every 15 minutes) that is more adequate to its spatial resolution (2.6 arcsec). Most space weather significant events last on the order of $30 \mathrm{~min}$ to $45 \mathrm{~min}$ : EIT waves (45 min), prominence eruptions (30 min) 
and flares (M-flare: $24 \mathrm{~min}, \mathrm{X}$-flare: $30 \mathrm{~min})^{7}$. This means that the EIT CME watch was sufficient to record the occurrence of these events, but not their temperature evolution (in the case of EIT waves or eruptions), or peak emission (in the case of flares). Thanks to this higher image cadence, SWAP will be a solar monitor capable of recording the time-evolution of every event in the low solar corona that might be of relevance to the space weather.

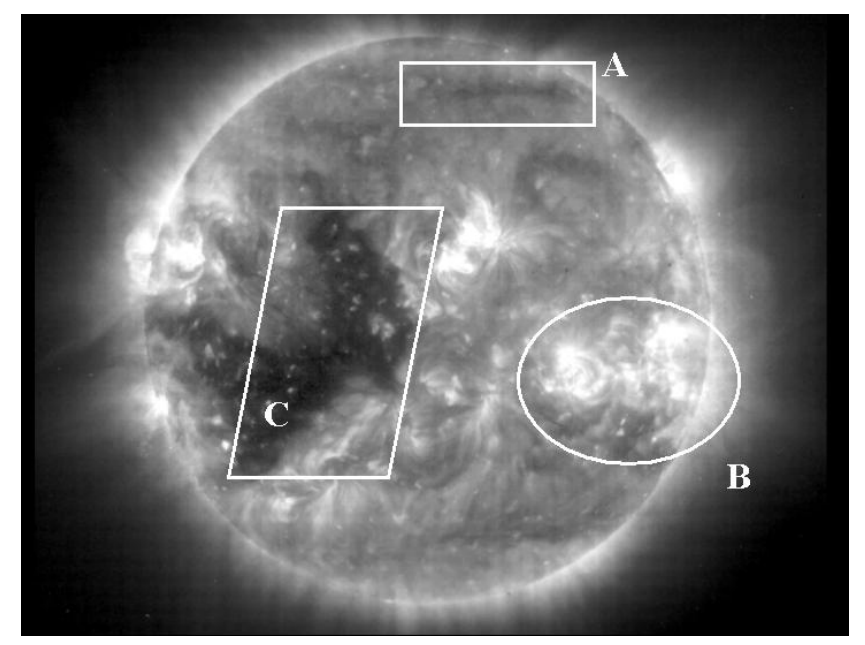

Figure 3: A $19.5 \mathrm{~nm}$ image taken by EIT on 2003/06/24 showing a filament channel (A), an active region (B) and a coronal hole (C). Since SWAP will be launched with the PROBA-2 spacecraft in 2006, close to solar minimum, the EUV corona will be simpler and more resembling the one shown in fig. 4 . Yet any of the features A,B, C will occasionally be present and able to drive space weather disturbances.

SWAP has a wide FOV, similar to EIT. However whereas EIT is mounted on large platform (SOHO) with fixed suncentred pointing, SWAP is mounted as the main instrument on a small platform. It is therefore in principle as flexible as, e.g., TRACE ${ }^{8}$, in changing its pointing. This opens opportunities for enlarging the FOV by paving the immediate neighbourhood of the Sun or to occasionally follow automatically eruptions as they lift off from the Sun. Joint observation campaigns with coronagraphs on other platforms could thus become possible.

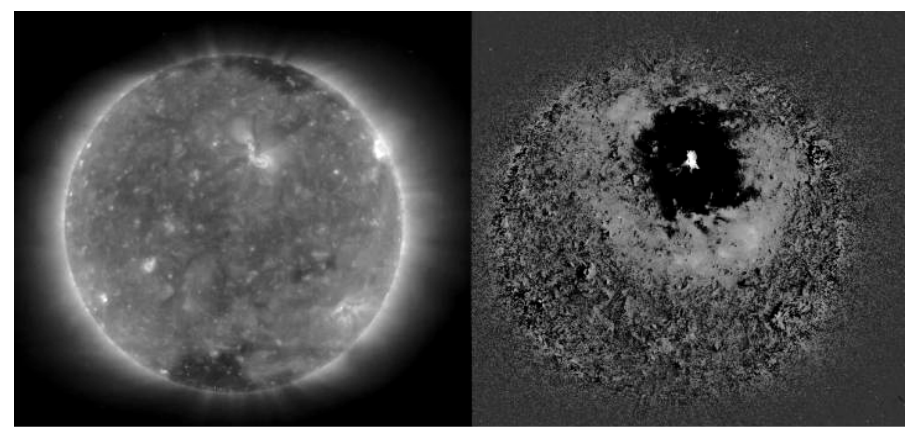

Figure 4: On the left, an EIT image in the Fe XII band pass $(19.5 \mathrm{~nm})$ taken on $1997 / 05 / 12$, just prior to a coronal mass ejection. On the right, a processed image showing clearly the EIT wave and coronal dimmings. This event was the earliest warning of the halo CME that later hit the Earth magnetosphere and produced an intense geomagnetic storm (peak Dst <100) two days and 22 hours later.

The APS and its associated smart electronics will not only improve the overall performance of the instrument. Through the non-destructive read-out (NDR), and random access of its pixels, fast changing regions will be sampled and monitored at a high cadence rate. This delivers for the first time temporal series of the highest interest for contextual flare to smaller brightenings studies.

The combination of shutterless (nominal) operations and NDR will naturally maximize the signal-to-noise ratio in the dimmest part of the image. With CCDs, the low signals are contaminated and made noisier by their (charge) transfer through brighter regions. Furthermore, the integration time is limited by the saturation of the first pixels. These two deleterious effects will not happen in SWAP thanks to its APS camera; this is particularly useful when off-pointing to the extended Corona. These new observations far above the limb require careful optical design of the stray-light rejection. If this operation scheme is implemented on PROBA-2, it is anticipated to overlap SWAP EUV observations with white-light coronagraph data to 2-6 solar radii, at least during CME passages.

An image processing pipeline will automatically detect the occurence of the above mentioned space weather related events in the stream of SWAP data. This pipeline will be implemented at the ground segment and will produce event catalogues and near real time warnings for space weather monitoning applications. In addition, it is also foreseen to run image recognition software onboard the spacecraft. This onboard module of the SWAP image recognition software will 
contribute to the demonstration of the autonomy of the PROBA-2 platform. The onboard module will target two applications: (1) prioritising the telemetry of images containing important space weather events and discarding others images, (2) commanding offpoints of the spacecraft in the direction of an erupting CME.

\section{INSTRUMENT DESCRIPTION}

\subsection{General description}

SWAP is based on a Ritchey-Chretien off-axis telescope, used to focus the EUV light from the solar corona on a CMOS detector. The key elements of the instrument are the optics and the focal plane assembly, which are outlined in the general scheme of the instrument illustrated in figure 5.

The SWAP observations cover a very narrow spectral range centred on $19.5 \mathrm{~nm}$ with a FWHM of the order of $\sim 1 \mathrm{~nm}$, in a field of view (FOV) of 45 arcmin. This spectral selection is performed with specific EUV multilayers deposited on the mirrors used in quasi-normal incidence. This specific coating is designed and optimized to cope with the off-axis angles and the stringent FWHM requirement. Additional light rejection will be provided by 2 aluminium filters $(\sim 150 \mathrm{~nm}$ thick), that will reject most of the visible and infrared solar radiation to avoid excessive heating of the mirrors. Those very fragile filters require specific care for handling and mounting in the instrument.

SWAP will provide images similar to the EIT $195 \AA$ channel, as per figure 4 left.

This new compact off-axis design brings several advantages that make the instrument compatible with the PROBA II platform:

- mirrors without central obstruction are smaller for a given area;

- $\quad$ smaller optical beam and small filters;

- $\quad$ off axis can allow compact baffling without high accuracy positioning;

- $\quad$ no need for an external baffle;

- $\quad$ small aperture thus large F\# and low sensitivity to defocus and thermal distortion;

- small aperture thus small cover mechanism.

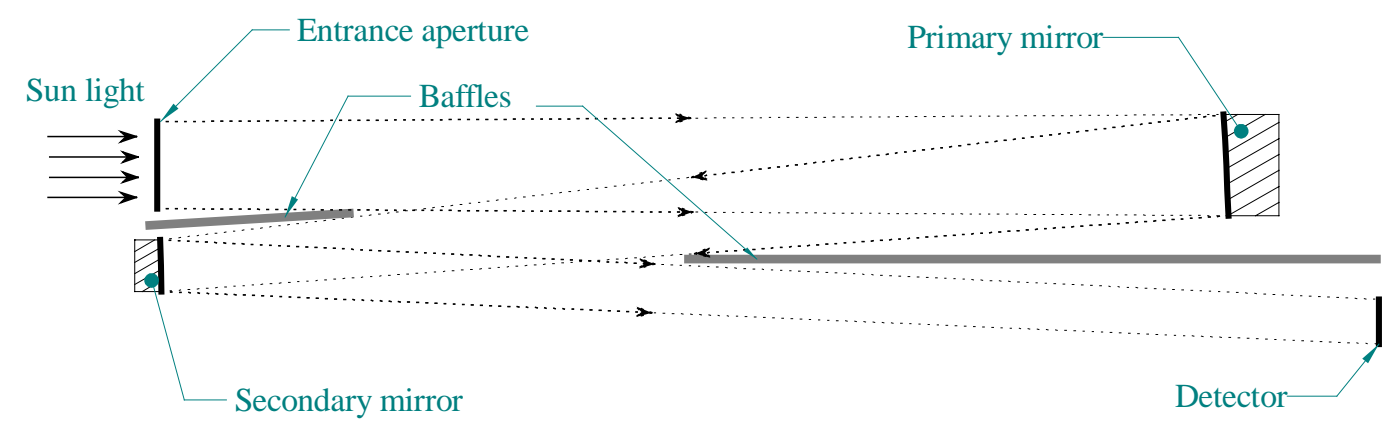

Figure 5: General concept of SWAP

The instrument concept is based on an optical baseplate, on which a 2-mirror telescope is mounted and aligned, to produce a focussed image on a detector.

The baseplate holds the following elements:

- a pair of mirrors;

- a focal plane assembly holding itself the detector, a set of calibration lamps and a radiator viewing cold space to cool the detector through a cold finger;

- a set of aluminium filters with an aperture stop; 
- $\quad$ a set of optical baffle (plane normal to the baseplate) to avoid unwanted straylight on the detector;

- $\quad$ an electronics unit to read the detector, drive and monitor the instrument;

- a one-shot opening aperture cover mechanism to protect the instrument during on-ground handling and launch ascent.

A preliminary design of the instrument is shown in figures $6,7 \& 8$.

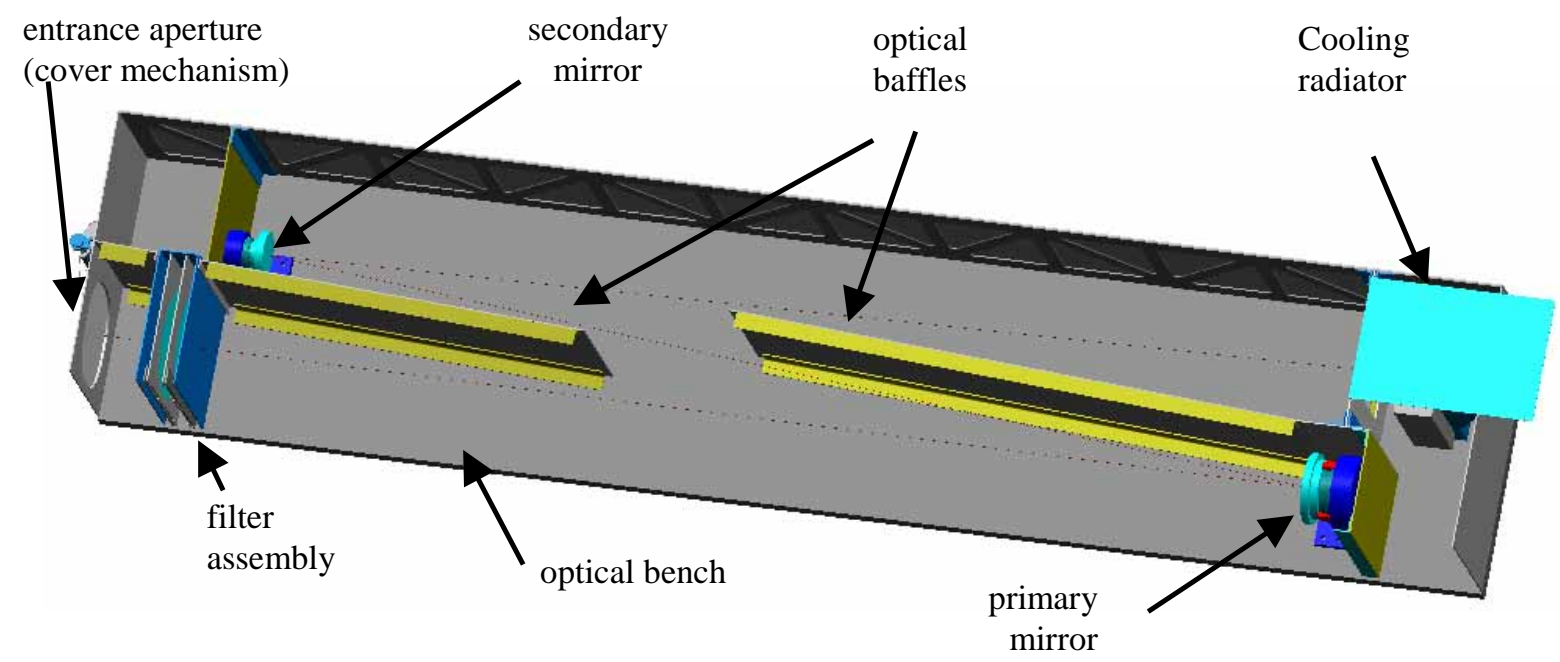

Figure 6: Open view of the SWAP instrument (the detector is hidden under the radiator)

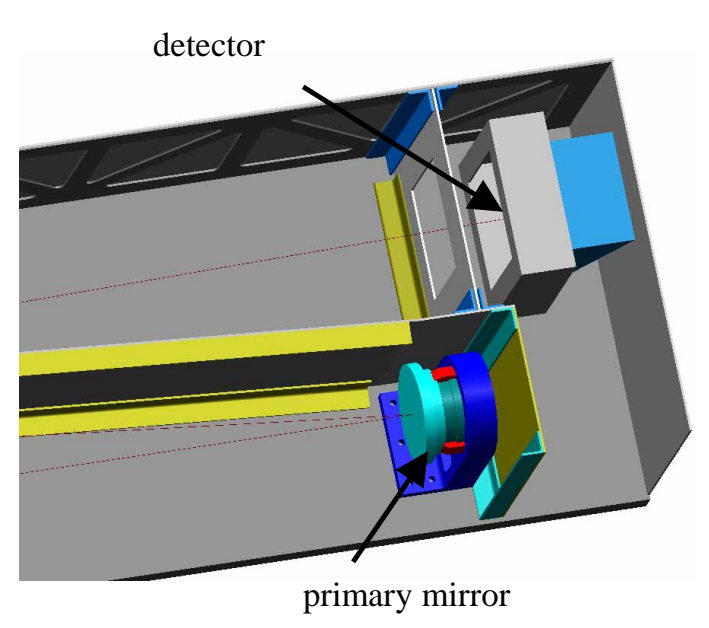

Figure 7: View of the rear section of SWAP

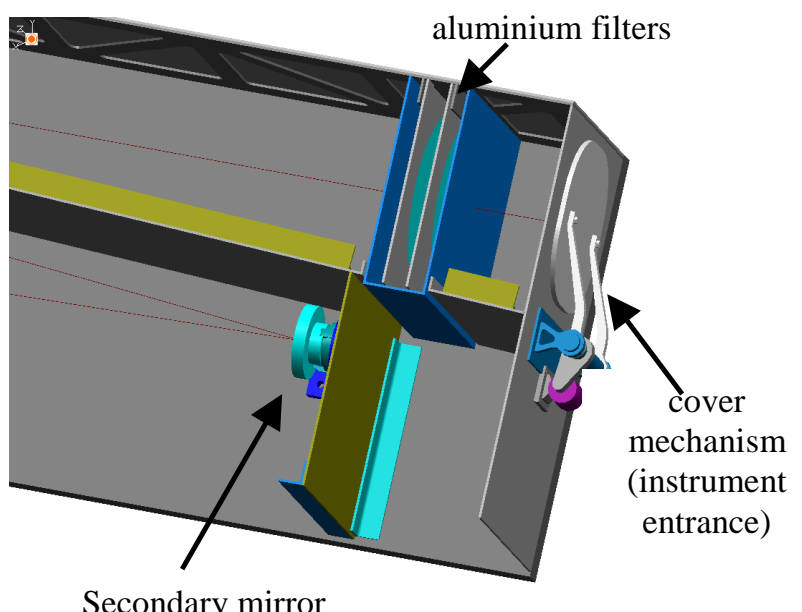

Secondary mirror
Figure 8: View of the front section of SWAP (rotated vs fig. 6)

\section{$3.2 \quad$ Optical Design}

The optical system has been designed with the parameters of table 1 . In order to cope with the restricted available volume and dimensions and to reduce the manufacturing cost of optical items, the design is based on a simplified off- 
axis Ritchey Chretien configuration. This design is derived from the optical scheme of SDO/MAGRITTE ${ }^{9}$, with adjustments for the SWAP detector. The off-axis allows to optimize the optical throughput with a minimum entrance aperture size (no central obscuration) which is required by the use of a front commercial LUXEL aluminium foil filter to reject the visible and IR light flux.

Two aluminium filters are required: one is mounted at the entrance pupil and the second close to the focal plane assembly. As shown in figure 9, to avoid a double pass into the front filter, the entrance pupil is located in the plane of the secondary mirror (diaphragm in fig. 9). This configuration limits the field aberrations as well as the design off-axis and by this way the incidence angle on multilayer coating on mirrors which makes the spectral rejection. The incidence angles on the mirrors are maintained below 6.2 arcdeg.

\begin{tabular}{|c|c|}
\hline Field of view: & 45 arcmin square \\
\hline $\begin{array}{c}\text { Spectral } \\
\text { wavelength: }\end{array}$ & $\begin{array}{c}195 \AA \text { central wavelength } \\
(1 \mathrm{~nm} \text { FWHM bandpass })\end{array}$ \\
\hline Entrance pupil: & $33 \mathrm{~mm}$ diameter \\
\hline Focal length: & $1173 \mathrm{~mm}$ \\
\hline Detector & $\begin{array}{c}\text { CMOS STAR } 1000 \\
1024 \times 1024 \text { pixels }-15 \mu \mathrm{m} \text { pitch }\end{array}$ \\
\hline Total track: & $<450 \mathrm{~mm}$ \\
\hline
\end{tabular}

Table 1: Design parameters

In order to simplify the manufacturing, the secondary mirror is made of a $26.5 \mathrm{~mm}$ diameter sphere. The $44 \mathrm{~mm}$ diameter primary mirror is tilted to compensate the aberrations. The primary mirror aspherisation is small and allows a direct interferometric measurement without costly null lens.

The off-axis configurations allows an efficient and simple baffling system without needing an external front baffle that would have been out of the total track requirement. The baffling system is composed by 2 simple planar baffles, one from the secondary mirror going towards the primary mirror and the second from the primary mirror going towards the secondary mirror. It prevents any direct straylight propagation from the entrance pupil towards the focal plane.

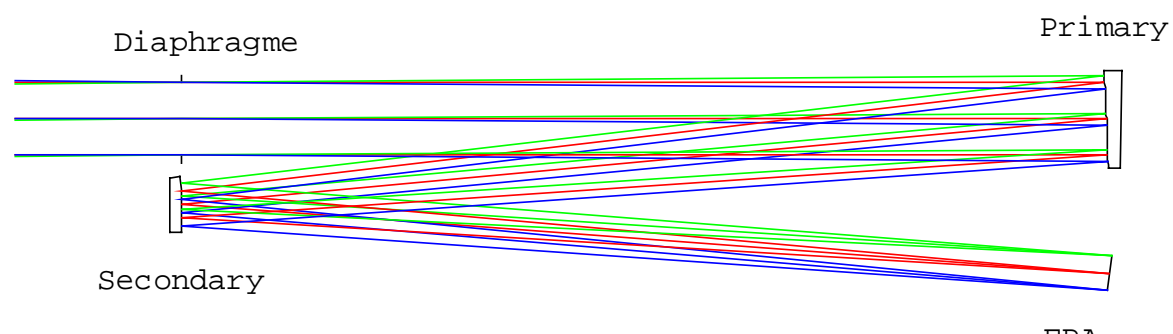

Figure 9: Optical design

\subsection{EUV Multilayers}

In order to achieve the required instrument throughput, both primary and secondary mirrors will be covered by multilayer coatings optimized for $19.5 \mathrm{~nm}$ high reflectivity.

Mo and Si materials have been chosen both for their optical and chemico-physical properties. The optical contrast of their refraction indexes guarantees a high reflection coefficient at each single interface; their low miscibility and limited chemical reaction at interfaces provide the necessary stability in time of the layers structure. These properties have been already verified with the EIT solar instrument ${ }^{4}$ on board of the SOHO platform. That instrument, a Ritchey-Chretien telescope with two Mo/Si multilayer coated mirrors ${ }^{10}$, is acquiring images of the full sun disk in the EUV spectral region since 1995. 
Mo/Si parameters optimization has been performed according to SWAP optical design and mirrors specifications. The incidence angle map on mirror M1 and M2, as the one reported in fig. 10, have been considered. The mean incidence angles have been calculated on both mirrors, and multilayer parameters have been optimized in order to have the spectral reflectivity peak at $19.5 \mathrm{~nm}$ for those incidence angles.

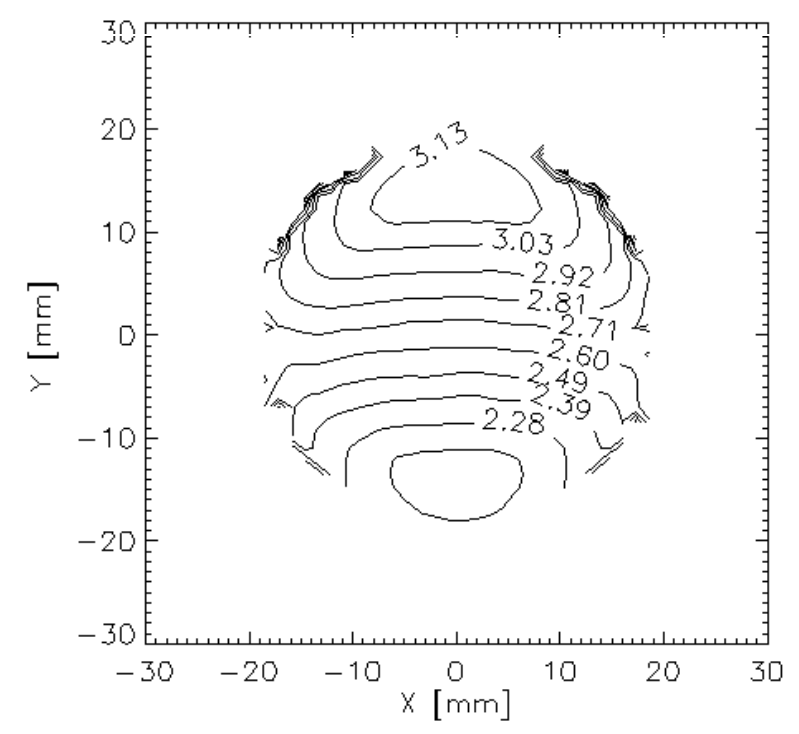

Figure 10: Incidence angle contour plot of mirror M1 (arcdeg)

Due to similar incidence angle distribution values, structural multilayer parameters for mirror M1 and M2 have been assumed to be the same (tab. 2). As a cap-layer, $15 \AA$ of Si will be further deposited on top of the layer stack. Part of this top layer will naturally oxidize forming a stable native oxide protection of the structure.

Simulation of each multilayer response has been performed with IMD software. The reflectivity curve for an ideal multilayer (i.e. in absence of interface roughness and interlayer compounds) is reported in fig. 11.

\begin{tabular}{|l|l|l|}
\hline & \multicolumn{1}{|c|}{ M1 } & \multicolumn{1}{|c|}{ M2 } \\
\hline \hline Mean incidence angle & $2.5^{\circ}$ & $4.5^{\circ}$ \\
Incidence angle range & $2-3.5^{\circ}$ & $3.5^{\circ}-5^{\circ}$ \\
\hline Multilayer & & \\
Top layer & $\mathrm{a}-\mathrm{SiO}$ x $10 \AA$ & $\mathrm{a}-\mathrm{SiO}_{\mathrm{x}} 10 \AA$ \\
& $\mathrm{a}-\mathrm{Si} 5 \AA$ & $\mathrm{a}-\mathrm{Si} 5 \AA$ \\
$\mathrm{N}$ of layers & 30 & 30 \\
Period d & $101.5 \AA$ & $101.5 \AA$ \\
$\Gamma_{\mathrm{dMo} / \mathrm{d}}$ & 0.24 & 0.24 \\
\hline
\end{tabular}

Table 2: Multilayer coatings structural parameters

In order to verify the performances of the same multilayer structure at the different incidence angles on the mirror surfaces, simulations with extreme values of the incidence angle range have been performed for both mirrors. The results show that peak position and curve profile variations are negligible.

Theoretically, in order to calculate the final performances of the two-mirror system together, for each point source in the FoV, the $\mathrm{R}_{\lambda}{ }^{\mathrm{M} 1{ }^{\mathrm{M}} 2}$ reflectivity curve should be evaluated using the distribution $\mathrm{m}(\theta, \varphi)$ (number of rays with $\theta$ incidence angle on M1 and $\varphi$ on M2) according to the following formula: 
with

$$
\mathrm{R}_{\lambda}=\frac{\sum_{\mathrm{ij}} \mathrm{m}\left(\theta_{\mathrm{i}}, \varphi_{\mathrm{j}}\right) \cdot \mathrm{r}_{1 \lambda}\left(\theta_{i}\right) \cdot \mathrm{r}_{2 \lambda}\left(\varphi_{\mathrm{j}}\right)}{\sum_{\mathrm{ij}} \mathrm{m}\left(\theta_{\mathrm{i}}, \varphi_{\mathrm{j}}\right)}
$$

- $\quad m\left(\theta_{\mathrm{i}}, \varphi_{\mathrm{j}}\right)$ fraction of rays reflected by M1 with an angle $\theta_{\mathrm{i}}$ that has a $\varphi_{\mathrm{j}}$ incidence angle on M2

- $\quad r_{1 \lambda}$ : reflectivity of $\mathrm{M} 1$ for an incidence angle $\theta_{\mathrm{i}}$

- $\quad r_{2 \lambda}$ : reflectivity of M2 for an incidence angle $\varphi_{\mathrm{j}}$

Since the angular spread on both mirrors M1 and M2 in this design is very small, only two representative cases $r_{1 \lambda} * r_{2 \lambda}$ have been considered, without weighting for the $\mathrm{m}$ factor, and without considering each single FoV:

case $A: \theta=2^{\circ}, \varphi=3.5^{\circ}$

- $\quad$ case B: $\theta=3^{\circ}, \varphi=5^{\circ}$

The results of simulation are reported in fig. 12. These simulations reveal that the spectral shift of the bandpass is absolutely negligible and that the coating will perform uniformly over the whole field of view.

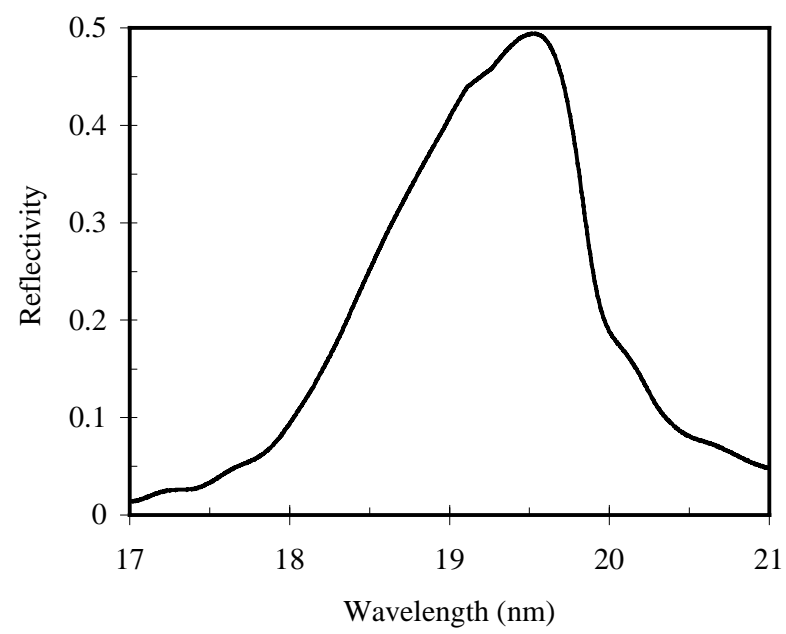

Figure 11: Multilayer theoretical reflectivity curve

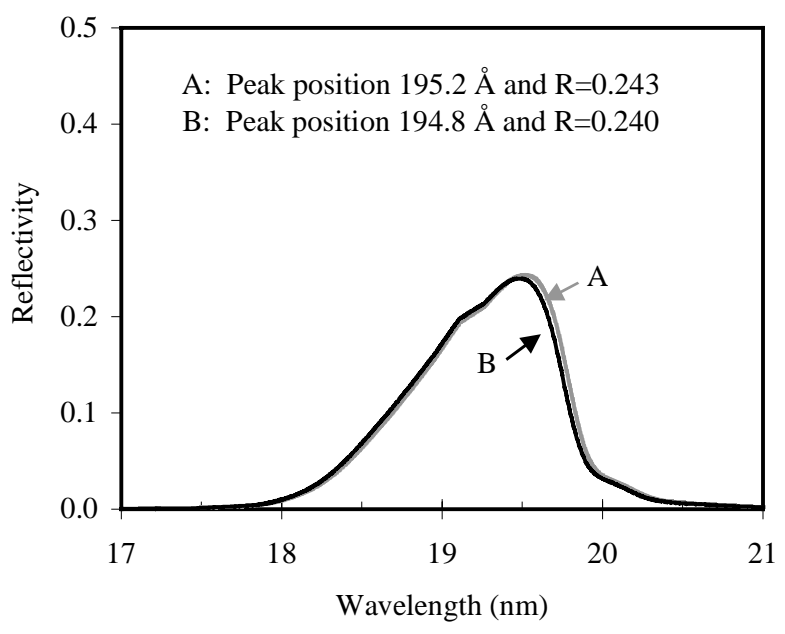

Figure 12: Theoretical reflectivity including the effects of the non-normal incidence (case A \& B)

Multilayer coatings ${ }^{11,12}$ will be deposited through magnetron sputtering techniques, in a $13.56 \mathrm{MHz}$ excited Ar plasma. The deposition system has the capability of depositing two $80 \mathrm{~mm}$ diameter samples in the same session. Standard nuclear and optical techniques, like RBS and XRR, will be used in order to optimize the process and check multilayer deposition.

Sample mirrors reflectivity measurements will be performed using a dedicated grazing incidence spherical grating monochromator located in the same area as the deposition facility, in order to provide feed-back on test sample deposition process. In order to perform a more detailed analysis of the multilayer performances (polarization dependence, scattering etc), some measurements will be performed at ELETTRA synchrotron beamline BEAR (Trieste, I).

\section{DETECTOR}

For SWAP the SWAP detector, the baseline is to use an existing APS-CMOS detector that will be supplied by FillFactory (B). Two candidate sensors are foreseen: STAR 250 with 512 x 512 pixels and STAR 1000, containing an array with 1024 by 1024 pixels at $15-\mu \mathrm{m}$ pitch. Both detectors include on-chip correction for Fixed Pattern Noise 
(FPN), a programmable gain output amplifier, and a 10-bit analog-to-digital converter (ADC), but they do not offer not NDR operation.

The so-called "well-pixel" architecture provides a fill factor close to $100 \%$ and a high charge to voltage conversion ${ }^{13}$. In addition, all circuits are designed to be radiation-hardened, limiting the degradation of the average dark current to less than $1 \mathrm{nA} / \mathrm{cm}^{2}$ after $3 \mathrm{Mrad}$ exposure.

In order to reduce noise level, the detector is cooled down to a temperature of $\sim-10^{\circ} \mathrm{C}$. This allows a dark current shot noise below 120 e- rms after 30s of integration time. With a readout noise of 54 e- rms per pixel, a signal to noise ratio close to 10 can be reached in the worst case of quiet sun regions radiance (see sec. 5.2)

The product Quantum Efficiency x Fill Factor is above $20 \%$ in the spectral range $450-750 \mathrm{~nm}$. In order to obtain a detection sensitivity in the EUV range, a specific enhancement is needed, based on the use of phosphor-like scintillator coating. A coating provided by Applied Scintillation Technologies (UK) will be evaluated in a first step, with two backup solutions already identified. The first one is Lumogen coating that was previously used on TRACE mission ${ }^{14}$. The second one is the Metachrome coating, offered by Roper Scientific.

Another option for SWAP detection is the use of the "next generation" APS detector, which are developed for future star tracker application in the scope of a European Space Agency (ESA) program. These sensors are still in the design phase, but the target performances foreseen for the High Accuracy Star tracker (HAS) detector show particular promises for an eventual application to SWAP configuration. Among the different advantages of the HAS, the following ones can be underlined:

- Dark current amplitude: the present level of STAR 1000 (around $470 \mathrm{e}-/ \mathrm{s}$ at-10 ${ }^{\circ} \mathrm{C}$ ) should be improved in the range 50 to $200 \mathrm{e}-/ \mathrm{s}$.

- Readout Noise: instead of having a readout noise at 54 e- (STAR 1000 level), this new detector would allow a noise level in the range $5 \sim 10$ e- rms.

- Efficiency: the sensor shall have a sensitivity (defined as Fill factor x Quantum Efficiency) of greater than $40 \%$ average over the visible spectral range.

- Possibility to operate in non destructive read-out (NDR) mode. Integration time can then be adjusted per pixel window.

The final choice for SWAP detection configuration will be done after experimental determination of scintillator layer efficiency in the EUV range, and confirmation of expected performances of HAS detector.

\section{INSTRUMENT PERFORMANCES}

\subsection{Optical performances}

Theoretical analyses were run to optimize and evaluate the resulting performance of the SWAP design. The RMS spot size is below 1.3 arcsec over a 45 arcmin circular field of view. The table 3 summarizes the expected RMS spot size degradation contributions, while the rms spot (design value) is shown in all the FOV in figure 13.

The optical performances are very dependent on the mirror manufacturing (i.e. wavefront error) and on the inter-mirror distance. On one hand, with a $\lambda / 20$ peak-to-peak surface error on both mirrors, the RMS spot size is degraded by 0.44 arcsec. On the other hand, with an inter-mirror distance variation lower than $\pm 50 \mu \mathrm{m}$, the RMS spot size degradation (diameter) is more than 0.63 arcsec. This requirement will be handled by the thermo-mechanical design, that can combine an aluminum structure with active thermal control, or a more temperature immune system requiring less power for thermal control.

The S/C jitter contribution has been evaluated with preliminary data, assuming an amplitude of 3 arcsec in the frequency range of 0.2 to $20 \mathrm{~Hz}$ and a 6 arcsec drift during exposure time (worst case, $60 \mathrm{~s}$ ). 


\begin{tabular}{|c|c|}
\hline Contributions & $\begin{array}{c}\text { RMS spot size } \\
\text { diameter } \\
\text { degradations }[\boldsymbol{\mu m}]\end{array}$ \\
\hline Design & $\begin{array}{c}7.5 \mu \mathrm{m} \text { at } 22.5 \\
\text { arcmin FOV }\end{array}$ \\
\hline Mirror figuring & $0.2 \mu \mathrm{m}$ \\
\hline $\begin{array}{c}\text { Mirror } \\
\text { irregularities }\end{array}$ & $2.5 \mu \mathrm{m}$ \\
\hline Alignment errors & $1.16 \mu \mathrm{m}$ \\
\hline Launch effects & $0.05 \mu \mathrm{m}$ \\
\hline In-flight conditions & $3.6 \mu \mathrm{m}$ \\
\hline S/C pointing & $6.46 \mu \mathrm{m}$ \\
\hline Total & $\begin{array}{c}\mathbf{1 5 . 3 8} \boldsymbol{\mu m} \\
(\sim \mathbf{2 . 7} \mathbf{~ a r c s e c})\end{array}$ \\
\hline \multicolumn{2}{|c}{}
\end{tabular}

Table 3: Summary of optical system tolerances

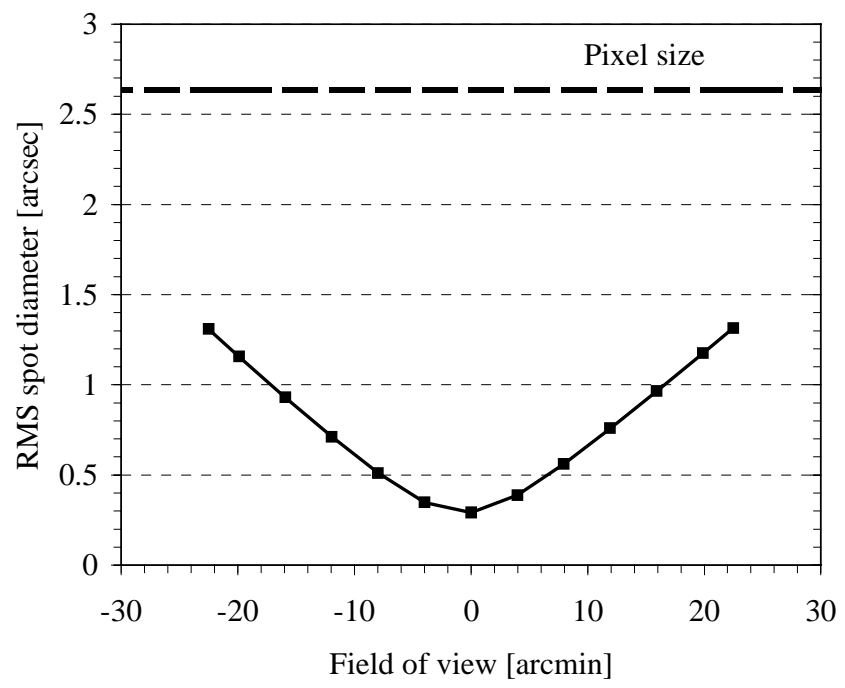

Figure 13: Spot diameter in the FOV of SWAP

\subsection{Instrument response}

The efficiency of the instrument is characterized by the signal to noise ratio, that will be evaluated in different signal conditions. A theoretical response model of the instrument has been built, including the response of all the optical and detector elements.

- The EUV mulitlayers have been modeled according to the results of the study summarized in fig. 12.

- The aluminum filter data are using Luxel filter properties for $1500 \AA$ aluminum layer, including the grid transmission $(82 \%)$ and an oxide layer.

- The scintillator quantum yield is taken $100 \%$, giving a scintillator efficiency of $50 \%$, since only half the visible photons are emitted towards the detector, the other half being emitted backwards.

- $\quad$ The detector sensitivities in the visible range are stated in paragraph 4.

The results of this model are summarized in fig. 14, showing the effective area, which is defined as the product of the optical efficiencies (geometric area, mirror reflectivity, filter transmission, and quantum efficiency).

This instrument response model has been convolved with the theoretical radiance levels at instrument entrance derived from the CHIANTI database ${ }^{\mathbf{1 5}}$. The signal-to-noise ratio (photon statistics) achieved after a 30-second exposure time, with both current STAR1000 detector and next generation HAS detector, is shown table 4. The results for the HAS detector option are based on conservative values (predicted thermal noise,...).

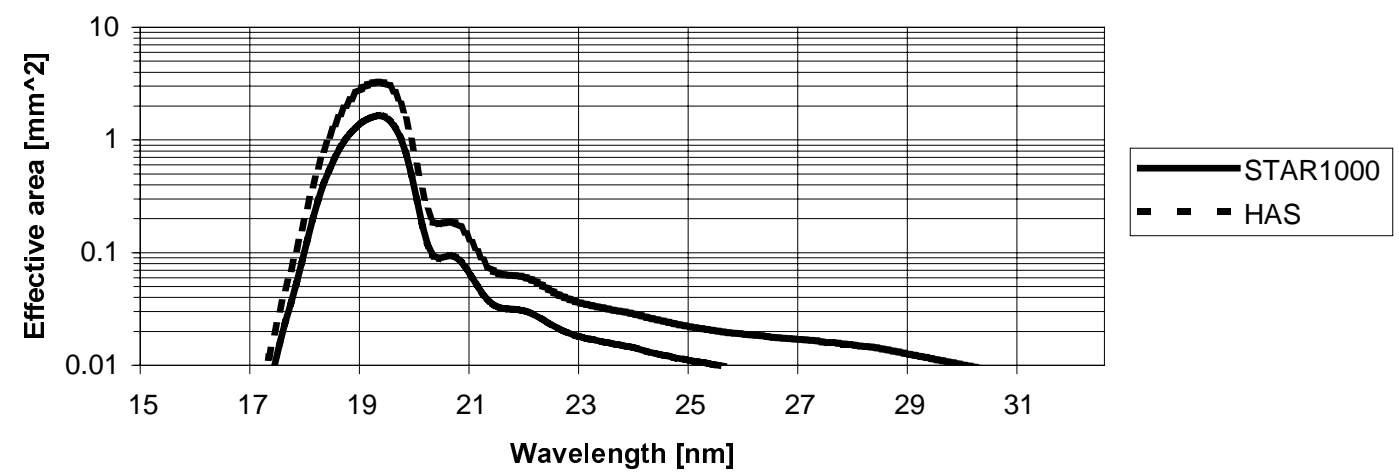

Figure 14: SWAP instrument wavelength selectivity 


\begin{tabular}{|l|c|c|c|}
\hline Detector & Effective Area $\left.\mathbf{( m m}^{\mathbf{2}}\right)$ & Quiet Sun S/N & Active Region S/N \\
\hline STAR1000 & 1.5 & 6.4 & 103 \\
\hline HAS & 3.0 & 18.3 & 183 \\
\hline
\end{tabular}

Table 4: SWAP signal to noise ratios (Quiet Sun and Active Regions correspond respectively to zones $\mathrm{C}$-dark regions- and $\mathrm{B}$-bright regions- of fig 3 )

The additional possibilities available with the readout of the HAS detector will allow different exposure times in different image areas, this will improve significantly the signal-to-noise ratio in the quiet Sun regions.

\section{CONCLUSIONS}

SWAP is a compact instrument of the $10 \mathrm{~kg}$-class, that will provide science observation for the solar physics community. Besides space weather observations, the SWAP instrument is also a technological demonstrator, using a novel compact off-axis optical design validating the optical concept of SDO/MAGRITTE. The instrument configuration provides also the possibility to integrate the recent developments performed in the field of CMOS detector technology, for the High Accuracy Star Tracker application. A preliminary assessment shows that the EUV enhancement of the detector is one of the most challenging aspect of the mission. Finally, SWAP will fully exploit the on-board autonomy philosophy, demonstrating image processing, data compression and filtering.

Future studies are planned to exploit the agility provided by the PROBA-2 platform. The off-pointing capability could allow a significant enlargement of the scientific field of view, with other important science outcomes. Design analyses will be conducted to analyse how to provide the adequate damping of the EUV straylight produced by the vicinity of the Sun close to the FOV during off-pointings.

\section{ACKNOWLEDGMENTS}

SWAP is developed by a consortium of international institutes: Centre Spatial de Liège (B), Royal Observatory of Belgium (B), University of Padova (I), LNL-INFN Group of Padova (I), and Max-Planck-Institut für Aeronomie (G). The Belgian contribution to the SWAP program is funded by the Federal Office for Scientific, Technical and Cultural Affairs (OSTC), in the frame of the ESA/Prodex program.

We wish to thank S.P. Airey from ESA/ESTEC for the communication of preliminary data concerning the HAS detector, and VERHAERT Design \& Development N.V. for providing advanced data on PROBA-2.

\section{REFERENCES}

1. P. Rochus, J.M. Defise, J.P. Halain, E. Mazy, R.A. Howard, J.D. Moses, J. Newmark, F. Clette, P. Cugnon, D. Berghmans, J.F. Hochedez, J.P. Delaboudinière, G. Artzner, F. Auchère, R. Mercier, M.F. Ravet, F. Delmotte, M. Idir, E. Antonucci, S. Fineschi, D. Gardiol, M. Romoli, M. Malvezzi, P. Nicolesi, G. Naletto, R.A. Harrison, U. Schühle; "MAGRITTE / SPECTRE : the Solar Atmospheric Imaging Assembly (AIA) aboard the Solar Dynamics Observatory", AGU Fall Meeting, Dec-2002.

2. P. Rochus, J.M. Defise, J.P. Halain, C. Jamar, E. Mazy, L. Rossi, T. Thibert, F. Clette, P. Cugnon, D. Berghmans, J.F. Hochedez, J.P. Delaboudinière, F. Auchère, R. Mercier, M.F. Ravet, F. Delmotte, M Idir, U. Schühle, V. Bothmer, S. Fineschi, R.A. Howard , J.D. Moses, J. Newmark, "MAGRITTE: an instrument suite for the Solar Atmospheric Imaging Assembly (AIA) aboard the Solar Dynamics Observatory”, Proc. SPIE 5171 (this volume), 2003.

3. F. Teston, R. Creasey, J. Bermyn, K. Mellab, "PROBA: ESA's Autonomy and Technology Demonstration Mission", Proceedings of the 13th Annual AIAA/USU, Conference on Small Satellites, August 23 - 26, 1999

4. JP. Delaboudinière, G.E. Artzner, J. Brunaud, A.H. Gabriel, J.F. Hochedez, F. Millier, X.Y. Song, B. Au, K.P. Dere, R.A. Howard, R. Kreplin, D. J. Michels, J.D. Moses, J.M. Defise, C. Jamar, P. Rochus, J.P. Chauvineau, J.P. Marioge, R.C. Catura, J.R. Lemen, L. Shing, R.A. Stern, J.B. Gurman, W.M. Neupert, A. Maucherat, F. Clette, P. Cugnon, E.L. Van Dessel., "EIT: Extreme-UV imaging telescope for the SOHO mission"; Solar Physics 162: 291312, 1995. 
5. ESWS_RAL-RP-0002 ver 10.0 pag. 57

6. Thompson, B. J.; Plunkett, S. P.; Gurman, J. B.; Newmark, J. S.; St. Cyr, O. C.; Michels, D. J. SOHO/EIT observations of an Earth-directed coronal mass ejection on May 12, 1997. GRL 25, No. 14, p. 2465 - 2468 (1998).

7. Veroniq, A. et al, “Temporal aspects and frequency distributions of solar soft X-ray flares”“,AA, 382, $1070,2002$.

8. K.T. Strong, M. Bruner, T. Tarbell, A. Title, and C.J. Wolfson, "TRACE - the Transition Region and Coronal Explorer," Space Sci. Rev., 70, 119-112, 1994.

9. E. Mazy, J.M. Defise, J.P. Halain, R. Mercier, M.F. Ravet, P. Rochus, L. Rossi, "MAGRITTE optomechanical design and mirror manufacturing", SPIE 5249, in preparation, 2003.

10. JP Chauvineau et al, "Description and performances of mirrors and multilayers for the extreme ultraviolet imaging telescope (EIT) of the SOHO mission", Proc. SPIE 1546, 576.

11.P. Nicolosi, A. Patelli, M.G. Pelizzo, V. Rigato, G. Maggioni, L. Depero, E. Bontempi, G. Mattei, L. Poletto, P. Mazzoldi, G Tondello, "First realization and characterization of multilayer EUV reflective coatings", in Soft X-Ray and EUV Imaging Systems I, D. A. Tichenor and J.A. Folta, eds., Proc. SPIE 4506, 76-83, (2001).

12. M.G. Pelizzo, A. Patelli, P. Nicolosi, , V. Rigato, G. Salmaso, E. Bontempi, D. Gardiol, "Development of multilayer coatings for solar space experiments", in Innovative Telescopes and Instrumentation for Solar Astrophysics, Proc. SPIE 4853, 381-392, (2003).

13. B. Dierickx, G. Meynants, D. Scheffer, "Near 100\% fill factor CMOS active pixels", in IEEE CCD \& AIS workshop, Brugge, Belgium, 5-7 june (1997); Proceedings p. P1

14. T.D. Tarbell, R.W. Nightingale, "Extreme Ultraviolet Flux and Sensitivity Changes in TRACE Images", AAS 200th meeting, Albuquerque, NM, June 2002.

15. K.P. Dere, E. Landi, H.E. Lanson, B.C. Monsignori Fossi, P.R. Young, “CHIANTI”, A\&A Supp., 125, $149,1997$. 